\title{
Mass spectral analysis of the multikinase inhibitor BZG and its metabolites and analysis of their binding to vascular endothelial growth factor receptor-2
}

\author{
Yan Lou ${ }^{1}$, Wenqi Qiu ${ }^{1}$, Zhe Wu ${ }^{1}$, Qian Wang ${ }^{1}$, Yunqing Qiu ${ }^{1}$, Su Zeng ${ }^{2}$ \\ ${ }^{1}$ State Key Laboratory for Diagnosis and Treatment of Infectious Disease, Collaborative Innovation Center for Diagnosis and \\ Treatment of Infectious Diseases, Key Laboratory of Precision Diagnosis and Treatment for Hepatobiliary and Pancreatic \\ Tumor of Zhejiang Province, The First Affiliated Hospital, Zhejiang University, Hangzhou, PR China \\ ${ }^{2}$ Laboratory of Pharmaceutical Analysis and Drug Metabolism, Zhejiang Province Key Laboratory of Anti-Cancer Drug \\ Research, College of Pharmaceutical Sciences, Zhejiang University, Hangzhou, PR China
}

Correspondence to: Yunqing Qiu, email: qiuyq@zju.edu.cn

Keywords: hepatocellular carcinoma, novel multikinase inhibitor, metabolite, VEGFR-2, eHiTS

Received: January 25, 2017 Accepted: March 08, 2017 Published: March 16, 2017

Copyright: Lou et al. This is an open-access article distributed under the terms of the Creative Commons Attribution License (CC-BY), which permits unrestricted use, distribution, and reproduction in any medium, provided the original author and source are credited.

\section{ABSTRACT}

We previously showed that BZG is a novel multitarget kinase inhibitor, which inhibited hepatocellular carcinoma in vivo and in vitro. In the present study, we used ultra-performance liquid chromatography coupled with quadrupole time-of-flight mass spectrometry (UPLC/Q-TOF MS) to characterize BZG and its metabolites generated in vivo. The probable metabolic mechanism was further confirmed by analysis of Phase I and Phase II metabolism in liver microsomes and with recombinant enzymes. In addition, the binding affinities of BZG metabolites to vascular endothelial growth factor receptor 2 (VEGFR2) were predicted using electronic high throughput screening (eHiTS). The results showed that BZG underwent phase I and phase II metabolism. We detected 11 BZG metabolites and identified hydroxylation, glucuronation, acetylation, sulfonation and degradation as the major metabolic processes in vivo and in vitro. Five of the eleven metabolites showed highly favorable eHiTS energy scores that were lower than sorafenib. Knowledge of the in vivo metabolic pathways of BZG and its binding affinities to VEGFR2 will be beneficial for further clinical development of BZG.

\section{INTRODUCTION}

Hepatocellular carcinoma (HCC) is the most common primary liver cancer and the third leading cause of cancer deaths worldwide [1]. Therapeutic options for advanced HCC are limited and in spite of aggressive local treatment, recurrence is very common [2]. Currently, sorafenib remains the only established systemic therapy that increases the overall survival of unresectable HCC patients by a few months [3]. Sorafenib is a multikinase inhibitor that blocks platelet-derived growth factor (PDGF), vascular endothelial growth factor (VEGF), c-kit and fibrosarcoma signaling [4]. Sorafenib has been the most effective drug in the clinical setting [2] and newer and effective drugs are necessary to clinically combat HCC.

In our previous studies, we designed a small library of compounds that were analogous to sorafenib and screened against multiple members of the tyrosine and serine/threonine protein kinase families. BZG was one of the novel inhibitors that potently and selectively inhibits activities of kinases including VEGFR, Flt3 (Fmslike tyrosine kinase), c-kit and other kinases similar to sorafenib $[5,6]$. BZG also inhibited in vitro proliferation of a panel of human cancer cells [7]. Moreover, our previous research indicated that BZG significantly inhibited Huh7 cell derived tumor xenografts in Balb/c nude mice $[7,8]$. Studies showed relatively high concentrations of BZG in the liver and kidney providing a pharmacokinetic (PK) basis for their effective use in hepatic and renal carcinomas [9]. However, the in vivo metabolism and probable drug-drug interactions of BZG are unclear.

Understanding drug metabolism is critical for druggability analysis. Drug metabolites are generally responsible for the bioactivity of the parent drug. In case of sorafenib, sorafenib $\mathrm{N}$-oxide is the major pharmacologically active metabolite that shows greater 
potency than sorafenib against VEGFR-2 [10-12]. Therefore, we investigated the anticancer activities of the BZG metabolites in this study.

$\mathrm{HCC}$ is a highly vascular tumor, which proliferates through angiogenesis mediated partly by VEGF and its multiple receptors including VEGFR2. VEGFR2 (also known as KDR or FLK1) is the primary receptor mediating the angiogenic activity of VEGF in distinct signal transduction pathways and regulates endothelial cell proliferation, migration, differentiation, and tube formation $[13,14]$. Since high VEGFR2 expression is associated with metastases and poor prognosis of HCC in preclinical and clinical studies, inhibition of angiogenesis is a potential therapeutic target [15].

The aim of this study was to elucidate their metabolic profiles of BZG and identify its metabolites by UPLC/Q-TOF MS method. Furthermore, we performed virtual high-throughput screening to investigate the binding affinities of BZG and its metabolites to the target receptor tyrosine kinase, VEGFR-2 using the eHiTS docking software.

\section{RESULTS}

\section{UPLC/ Q-TOF MS analysis of BZG}

The chromatographic and mass spectral fragmentation patterns of BZG were investigated by UPLC/Q-TOF MS (Figure 1). The protonated BZG at $\mathrm{m} / \mathrm{z} 447$ was eluted at a retention time of $12.26 \mathrm{~min}$. We observed product ions at $\mathrm{m} / \mathrm{z} 252,226,209,194$, and 134 (100\% abundance). The fragment ions at $\mathrm{m} / \mathrm{z} 252$ and $\mathrm{m} / \mathrm{z}$ 194 were generated by the cleavage of the $\mathrm{C}-\mathrm{N}$ bond of the protonated molecular ion. Further loss of CO (26Da) from the fragment ion at 252 generated the fragment ion at $\mathrm{m} / \mathrm{z} 226$ and its subsequent loss of $\mathrm{C}_{6} \mathrm{H}_{6} \mathrm{~N}(92 \mathrm{Da})$ resulted in the fragment ion at $\mathrm{m} / \mathrm{z} 134$. Based on the results obtained, we proposed the fragmentation pathway of BZG as shown in Figure 1B. The structure of BZG was divided into parts $\mathrm{A}, \mathrm{B}$, and $\mathrm{C}$ (Figure 1). These fragment ions were used as references to interpret the fragment ions of the metabolites and to examine the high resolution and mass accuracy of the instrument.

\section{Metabolic profile of BZG}

As shown in Figure 2, we detected 11 metabolites of BZG in vivo and in vitro. Table 1 lists the detailed information of these metabolites, including the retention times, proposed elemental compositions, and the characteristic fragment ions. The structures of metabolites were characterized based on mass spectral fragmentation patterns. Based on the metabolites identified, we concluded that the main metabolic pathways of BZG included hydroxylation, glucuronation, acetylation, sulfonation and degradation. The representative TOF MS spectra and their proposed fragmentation pathways are shown in Figure 3 and Figure 4.

\section{Identification and characterization of BZG metabolites generated in vivo}

After oral administration of BZG, 6 metabolites were obtained in the fecal samples (M1-M6) and 4 metabolites were found in bile samples (M1, M2, M5 and M6). In the urine and plasma samples, only the parent compound was detected.

\section{Metabolite M1 is generated by hydroxylation of BZG}

The M1 metabolites were eluted at retention time of $12.16 \mathrm{~min}$ and showed a protonated molecular ion at $\mathrm{m} / \mathrm{z}$ 463 , which was $16 \mathrm{Da}$ higher than the protonated BZG ion at $\mathrm{m} / \mathrm{z} 447$, suggesting addition of a single oxygen atom to BZG. The major fragment ions of M1 were at $\mathrm{m} / \mathrm{z} 268$, $242,225,194,150$. Moreover, the fragment ions at $\mathrm{m} / \mathrm{z}$ $268,242,225,150$ were $16 \mathrm{Da}$ higher than the fragment ions at $\mathrm{m} / \mathrm{z} 252,226,209,134$ of the parent compound, respectively, implying that the addition of the single oxygen atom occurred in the part $\mathrm{C}$. The fragment ion at $\mathrm{m} / \mathrm{z} 194$ was the same as the characteristic fragment peak of BZG, indicating that part A was intact. Based on these observations, we concluded that the M1 metabolite was generated by hydroxylation of BZG in part $\mathrm{C}$.

\section{M2, M3 and M4 metabolites are generated by glucuronidation and degradation of BZG}

The metabolite M2 eluted at a retention time of 1.82 min (Figure 2). It contained a protonated molecular ion at $\mathrm{m} / \mathrm{z} 471$, which was $24 \mathrm{Da}$ higher than protonated BZG ion at $\mathrm{m} / \mathrm{z} 447$. The main fragment products were at $\mathrm{m} / \mathrm{z}$ 295,261 , and 175. Loss of glucuronic acid (176Da) from the protonated ion generated the major fragment ion at $\mathrm{m} / \mathrm{z}$ 295 , which further lost fluoride and oxygen (35Da) to form the fragment ion at $\mathrm{m} / \mathrm{z} 261$. The fragment ion at $\mathrm{m} / \mathrm{z} 175$ indicated the presence of the glucuronide residue in the structure of part B. Therefore, M2 was a monoglucuronide conjugate of BZG with removal of fluoride and part $\mathrm{C}$.

M3 had a retention time of $1.50 \mathrm{~min}$ and was a protonated molecule at $\mathrm{m} / \mathrm{z} 579$. It had the elemental composition of $\mathrm{C}_{23} \mathrm{H}_{24} \mathrm{~N}_{4} \mathrm{O}_{14}$, suggesting its generation by addition of $\mathrm{C}_{12} \mathrm{H}_{16} \mathrm{O}_{12}$ (glucuronidation) and loss of part A from BZG. The high energy mass spectrum of M3 showed fragment ions at $\mathrm{m} / \mathrm{z} 109,249,285,294$, and 403. The fragment ion at $\mathrm{m} / \mathrm{z} 403$ was due to a loss of $176 \mathrm{Da}$ from the protonated ion, followed by the loss of $\mathrm{C}_{6} \mathrm{H}_{5} \mathrm{O}_{2}(109 \mathrm{Da})$ to generate the fragment ion at $\mathrm{m} / \mathrm{z} 294$. The fragment ion at $\mathrm{m} / \mathrm{z} 294$ further lost $\mathrm{COOH}$ (45Da) to form the fragment ion at $\mathrm{m} / \mathrm{z} 249$. In addition, the major fragment ion at $\mathrm{m} / \mathrm{z} 285$ was formed by the cleavage of 
Table 1: Identification of BZG metabolites in vivo and in vitro using UPLC/Q-TOF MS mass spectrometry

\begin{tabular}{|c|c|c|c|c|c|c|c|c|c|c|}
\hline Metabolite & Description & $\begin{array}{l}\text { Retention } \\
\text { time (min) }\end{array}$ & Formula & $\begin{array}{l}\text { Measured } \\
\text { mass [M-H]- }\end{array}$ & $\begin{array}{l}\text { Calculated } \\
\text { mass }[\mathrm{M}-\mathrm{H}]-\end{array}$ & Fragment ions & $\begin{array}{l}\text { Found } \\
\text { in feces }\end{array}$ & $\begin{array}{l}\text { Found } \\
\text { in bile }\end{array}$ & $\begin{array}{l}\text { Found in } \\
\text { urine }\end{array}$ & $\begin{array}{l}\text { Found in } \\
\text { plasma }\end{array}$ \\
\hline M1 & hydroxylation & 12.16 & $\mathrm{C}_{19} \mathrm{H}_{12} \mathrm{ClF}_{3} \mathrm{~N}_{6} \mathrm{O}_{3}$ & 463.0533 & 463.0533 & $\begin{array}{l}150,194,225,242 \\
268\end{array}$ & $\mathrm{Y}$ & $\mathrm{Y}$ & $\mathrm{N}$ & $\mathrm{N}$ \\
\hline M7 & hydroxylation & 11.00 & $\mathrm{C}_{19} \mathrm{H}_{12} \mathrm{ClF}_{3} \mathrm{~N}_{6} \mathrm{O}_{3}$ & 463.0530 & 463.0533 & $\begin{array}{l}134,175,191,194, \\
210,238,252,\end{array}$ & $\mathrm{~N}$ & $\mathrm{~N}$ & $\mathrm{~N}$ & $\mathrm{~N}$ \\
\hline M8 & hydroxylation & 11.49 & $\mathrm{C}_{19} \mathrm{H}_{12} \mathrm{ClF}_{3} \mathrm{~N}_{6} \mathrm{O}_{3}$ & 463.0531 & 463.0533 & $\begin{array}{l}150,194,225,242 \\
268\end{array}$ & $\mathrm{~N}$ & $\mathrm{~N}$ & $\mathrm{~N}$ & $\mathrm{~N}$ \\
\hline M2 & degradation and glucuronidation & 1.85 & $\mathrm{C}_{20} \mathrm{H}_{19} \mathrm{~F}_{3} \mathrm{~N}_{2} \mathrm{O}_{8}$ & 471.1064 & 471.1015 & $175,261,295$ & $\mathrm{Y}$ & $\mathrm{Y}$ & $\mathrm{N}$ & $\mathrm{N}$ \\
\hline M3 & degradation and glucuronidation & 1.50 & $\mathrm{C}_{23} \mathrm{H}_{24} \mathrm{~N}_{4} \mathrm{O}_{14}$ & 579.1163 & 579.1211 & $\begin{array}{l}109,249,285,294 \\
403\end{array}$ & $\mathrm{Y}$ & $\mathrm{N}$ & $\mathrm{N}$ & $\mathrm{N}$ \\
\hline M4 & degradation and glucuronidation & 2.43 & $\mathrm{C}_{13} \mathrm{H}_{14} \mathrm{~F}_{3} \mathrm{NO}_{6}$ & 336.0723 & 336.0695 & $59,75,89,160,175$ & $\mathrm{Y}$ & $\mathrm{N}$ & $\mathrm{N}$ & $\mathrm{N}$ \\
\hline M5 & degradation and sulfonation & 2.66 & $\mathrm{C}_{7} \mathrm{H}_{6} \mathrm{~F}_{3} \mathrm{NO}_{3} \mathrm{~S}$ & 239.9972 & 239.9942 & 80,160 & $\mathrm{Y}$ & $\mathrm{Y}$ & $\mathrm{N}$ & $\mathrm{N}$ \\
\hline M6 & degradation and acetylation & 3.02 & $\mathrm{C}_{16} \mathrm{H}_{13} \mathrm{~N}_{5} \mathrm{O}_{4}$ & 338.0874 & 338.0889 & $\begin{array}{l}59,117,134,162, \\
175\end{array}$ & $\mathrm{Y}$ & $\mathrm{Y}$ & $\mathrm{N}$ & $\mathrm{N}$ \\
\hline M9 & glucuronidation & 7.40 & $\mathrm{C}_{25} \mathrm{H}_{20} \mathrm{ClF}_{3} \mathrm{~N}_{6} \mathrm{O}_{8}$ & 623.0907 & 623.0905 & $\begin{array}{l}59,73,101,103, \\
179,194,222,252\end{array}$ & $\mathrm{~N}$ & $\mathrm{~N}$ & $\mathrm{~N}$ & $\mathrm{~N}$ \\
\hline M10 & glucuronidation & 9.92 & $\mathrm{C}_{25} \mathrm{H}_{20} \mathrm{ClF}_{3} \mathrm{~N}_{6} \mathrm{O}_{8}$ & 623.0904 & 623.0905 & $226,252,404$ & $\mathrm{~N}$ & $\mathrm{~N}$ & $\mathrm{~N}$ & $\mathrm{~N}$ \\
\hline M11 & glucuronidation & 10.75 & $\mathrm{C}_{25} \mathrm{H}_{20} \mathrm{ClF}_{3} \mathrm{~N}_{6} \mathrm{O}_{8}$ & 623.0907 & 623.0905 & $\begin{array}{l}134,194,252,402 \\
428,447\end{array}$ & $\mathrm{~N}$ & $\mathrm{~N}$ & $\mathrm{~N}$ & $\mathrm{~N}$ \\
\hline
\end{tabular}
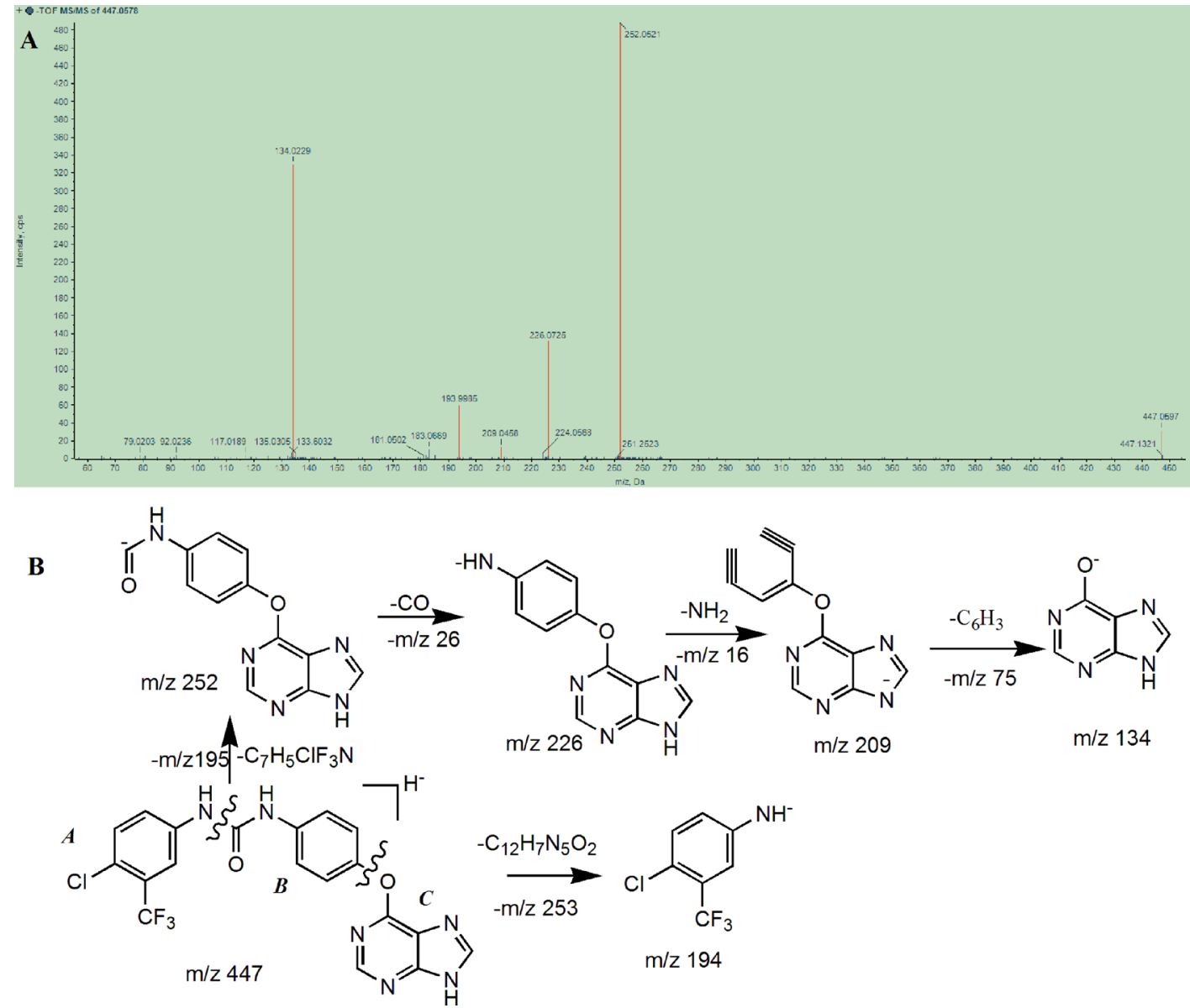

Figure 1: (A) Mass spectrum of BZG obtained on Q-TOF mass spectrometry and (B) Tentative structures of the most informative fragment ions for BZG. 
the $\mathrm{C}-\mathrm{O}$ bond of the protonated ion followed by loss of $\mathrm{C}_{6} \mathrm{H}_{8} \mathrm{O}_{6}(176 \mathrm{Da})$ to form the fragment ion at $\mathrm{m} / \mathrm{z} 109$. This indicated the presence of another glucuronide residue in the structure.

Metabolite M4 was eluted at a retention time of 2.43 min, showing a protonated molecular ion at $\mathrm{m} / \mathrm{z} 336$. The major fragment product at $\mathrm{m} / \mathrm{z} 160$ was due to a neutral loss of 176Da from the parent BZG ion. Moreover, the characteristic fragment peaks of glucuronic acid at $\mathrm{m} / \mathrm{z} 59$, 75, 89 and 175 further indicated that the M4 metabolite was a result of glucuronidation and degradation of BZG.

\section{M5 metabolite is generated by sulfonation and degradation of BZG}

The metabolite M5 showed an UPLC elution profile with a retention time at $2.66 \mathrm{~min}$. The protonated molecular ion at m/z 240 was a degradation product since it was 231Da lower than BZG. The major fragment ions of M5 were $\mathrm{m} / \mathrm{z} 80$ and 160 . The fragment ion at $\mathrm{m} / \mathrm{z} 80$ was characteristic of sulfonic acid. The fragment ion at $\mathrm{m} / \mathrm{z} 160$ was formed by the loss of $\mathrm{SO}_{3} \mathrm{H}(80 \mathrm{Da})$ from the precursor ion at $\mathrm{m} / \mathrm{z} 240$ that originated from a modified NH portion. Therefore, we concluded that M5 was generated by sulfonation of BZG and removal of parts B and C.

\section{M6 metabolite is generated by acetylation and degradation of BZG}

Metabolite M6 had a retention time of $3.02 \mathrm{~min}$. It had a protonated molecular weight of $338 \mathrm{Da}$, which was 109Da lower than that of protonated BZG. The major fragment ions of M6 were at m/z 175, 162, 134, 117 and 59. The fragment ions at $\mathrm{m} / \mathrm{z} 175$ and 162

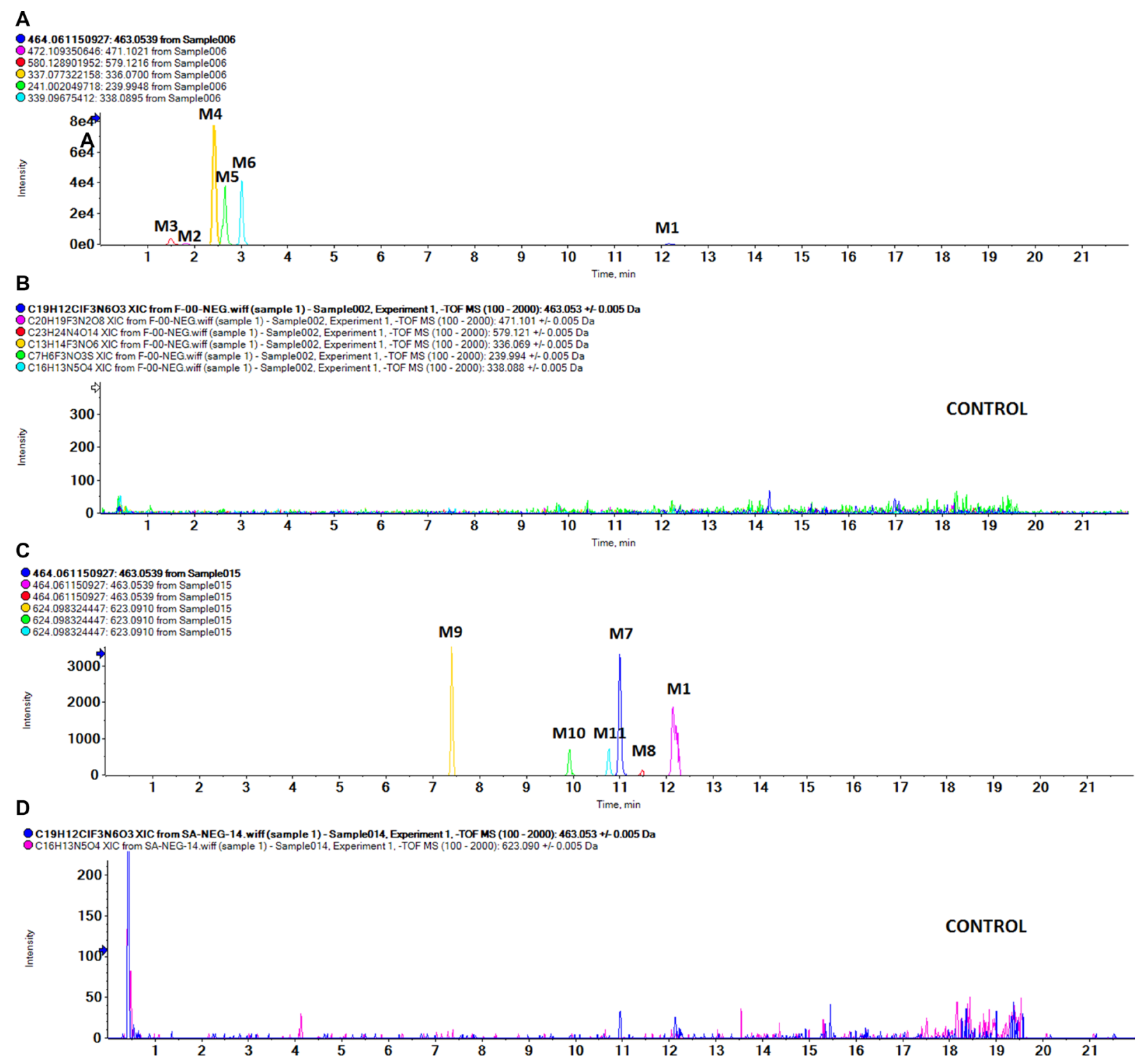

Figure 2: UPLC/MS chromatograms of BZG and its metabolites. (A) In vivo sample; (B) Blank sample; (C) Phase I and Phase II metabolism in liver microsomes; (D) Control sample. 
were generated by the cleavage of the $\mathrm{C}-\mathrm{O}$ bond of the protonated molecular ion at $\mathrm{m} / \mathrm{z} 338$. The fragment ion of $\mathrm{m} / \mathrm{z} 175$ was formed by the loss of $\mathrm{C}_{2} \mathrm{HO}$ (41Da) from characteristic fragment ion of BZG $(\mathrm{m} / \mathrm{z} 134)$, revealing acetylation on part $\mathrm{C}$. Then, this fragment ion lost $\mathrm{CH}_{2} \mathrm{CO}$ and oxygen atom to form the fragment ion at $\mathrm{m} / \mathrm{z} 117$. The fragment ion at $\mathrm{m} / \mathrm{z} 162$ lost a CO to form the ion at $\mathrm{m} / \mathrm{z} 134$, which further lost the benzene ring to form the fragment ion at $\mathrm{m} / \mathrm{z} 59$, indicating another acetylation event. Therefore, M6 was degradation result of acetylation in parts $\mathrm{B}$ and $\mathrm{C}$ of $\mathrm{BZG}$ and further degradation.

\section{Identification of in vitro BZG metabolites}

\section{Metabolism of BZG in human liver microsomes (HLMs)}

Compared with the control sample, 3 oxidative metabolites (M1, M7, and M8) were obtained in Phase I metabolism of BZG. In addition, 3 monoglucuronide conjugates of BZG (M9-M11) were detected in Phase II metabolism of BZG.

\section{M7 and M8 metabolites are generated by hydroxylation of BZG}

Metabolites M7 and M8 were eluted at retention times of 11.00 and $11.49 \mathrm{~min}$, respectively. Both showed a protonated molecular ion at $\mathrm{m} / \mathrm{z} 463$, which was $16 \mathrm{Da}$ higher than that at $\mathrm{m} / \mathrm{z} 447$ suggesting addition of a single oxygen atom. The major fragmentation of $M 7$ was at $\mathrm{m} / \mathrm{z}$ 210 , which was $16 \mathrm{Da}$ higher than the fragment ion at $\mathrm{m} / \mathrm{z}$ 194 of the parent BZG, implying that the modification was in part $\mathrm{C}$. This fragment ion further lost either a fluorine (19Da) or a chlorine atom (36Da) to form fragment ions at $\mathrm{m} / \mathrm{z} 191$ and 175, respectively. The fragment ion at $\mathrm{m} / \mathrm{z} 238$ was generated by the addition of $\mathrm{CO}_{2}(44 \mathrm{Da})$ to the ion at $\mathrm{m} / \mathrm{z} 194$. Moreover, the fragment ions at $\mathrm{m} / \mathrm{z}$ 252 and 134 indicated that parts $\mathrm{B}$ and $\mathrm{C}$ were intact. The metabolite M8 had similar fragment ions as M1, suggesting that the two metabolites were isomers. Based on these observations, we concluded that M7 and M8 were generated by hydroxylation of BZG in parts A and $\mathrm{C}$, respectively. However, the exact sites of hydroxylation could not be characterized.

\section{M9, M10 and M11 metabolites are generated by glucuronidation of BZG}

The BZG metabolites M9, M10 and M11 were eluted at retention times of 7.40, 9.92 and $10.75 \mathrm{~min}$, respectively. All the three metabolites showed a protonated molecular ion at $\mathrm{m} / \mathrm{z} 623$. The elemental composition of this metabolite was $\mathrm{C}_{25} \mathrm{H}_{20} \mathrm{ClF}_{3} \mathrm{~N}_{6} \mathrm{O}_{8}$, corresponding to the monoglucuronide conjugate of $\mathrm{BZG}$.

The fragment ions of M9 were observed at $\mathrm{m} / \mathrm{z} 59$, $73,101,103,179,194,222$, and 252 . The fragment ions

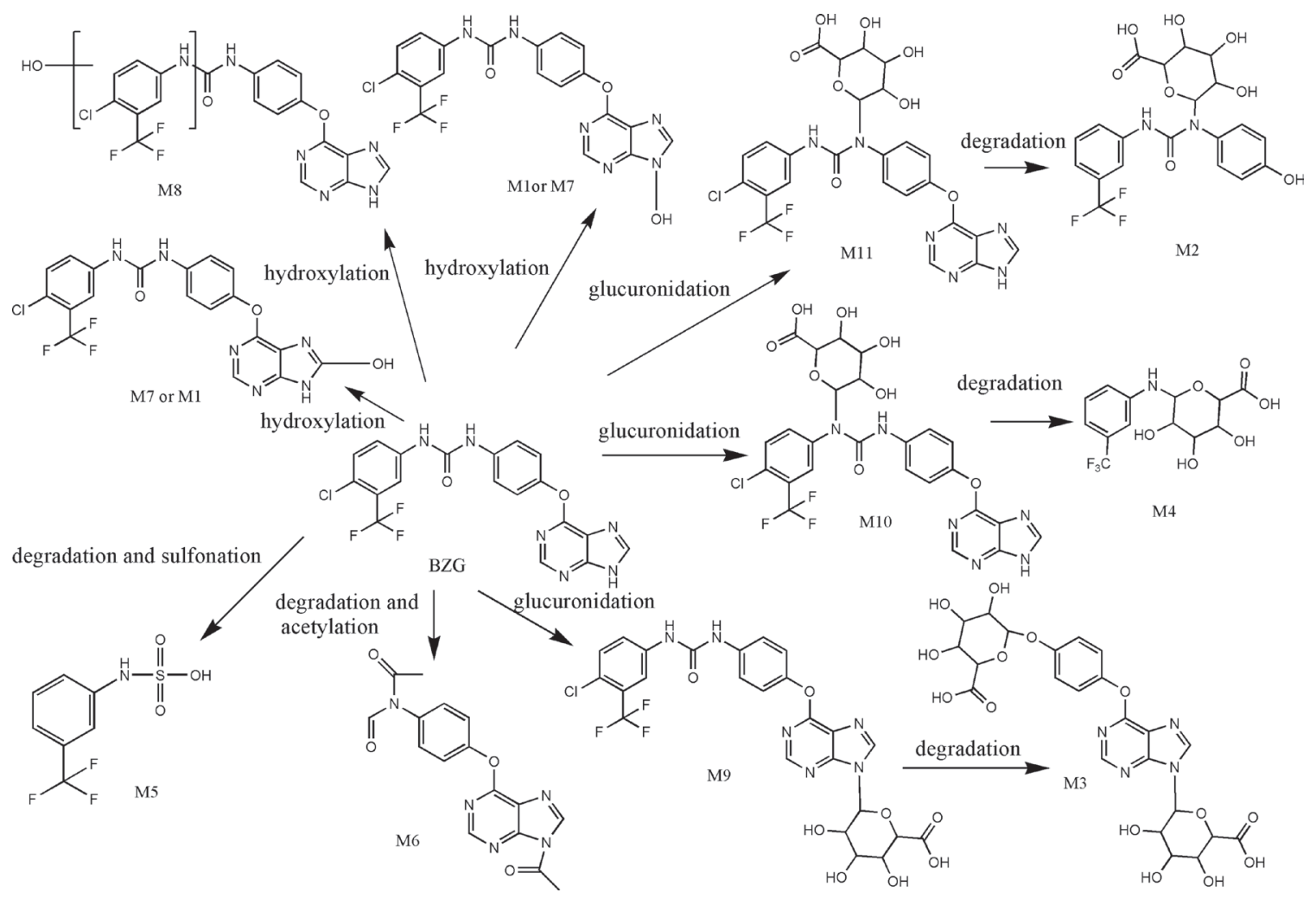

Figure 3: Proposed in vivo and in vitro metabolic pathways of BZG. 

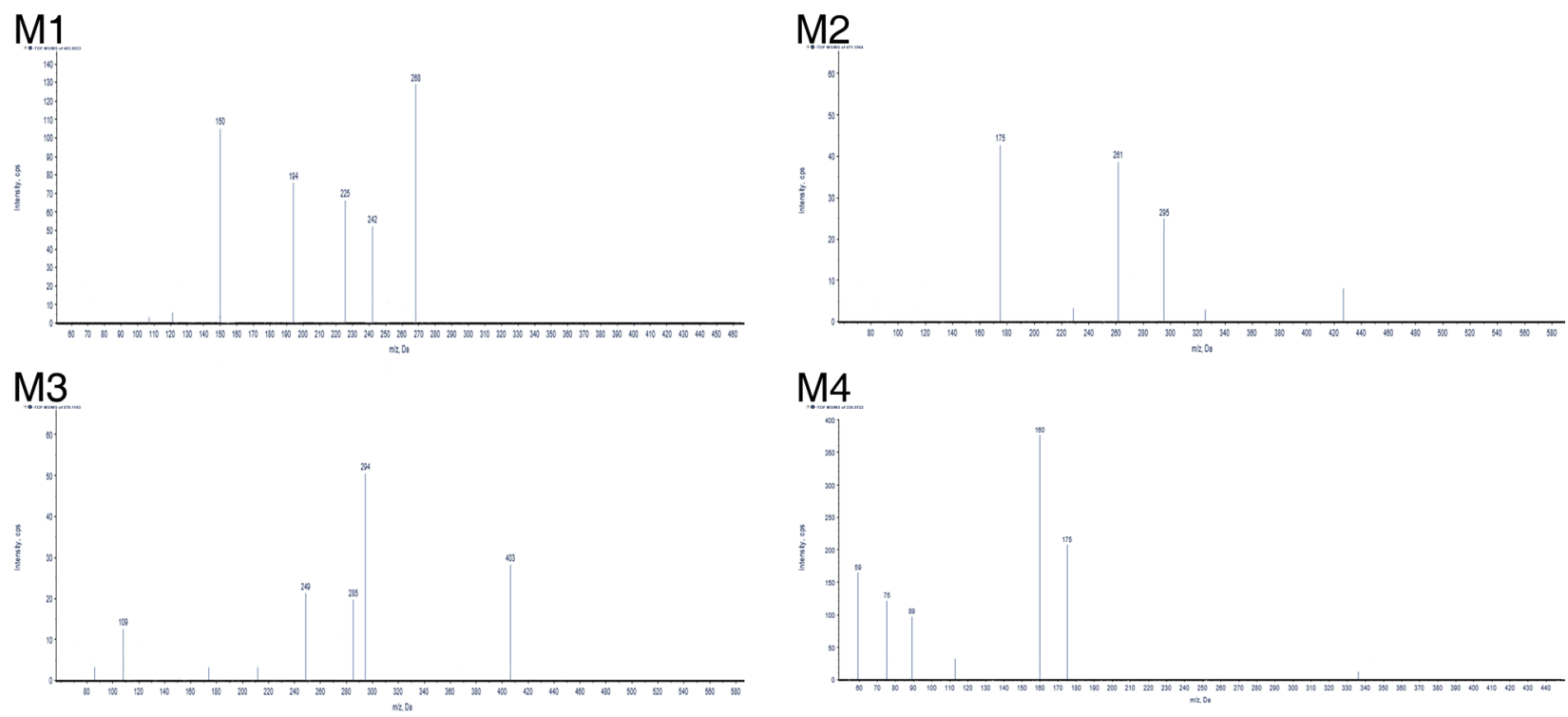

M4
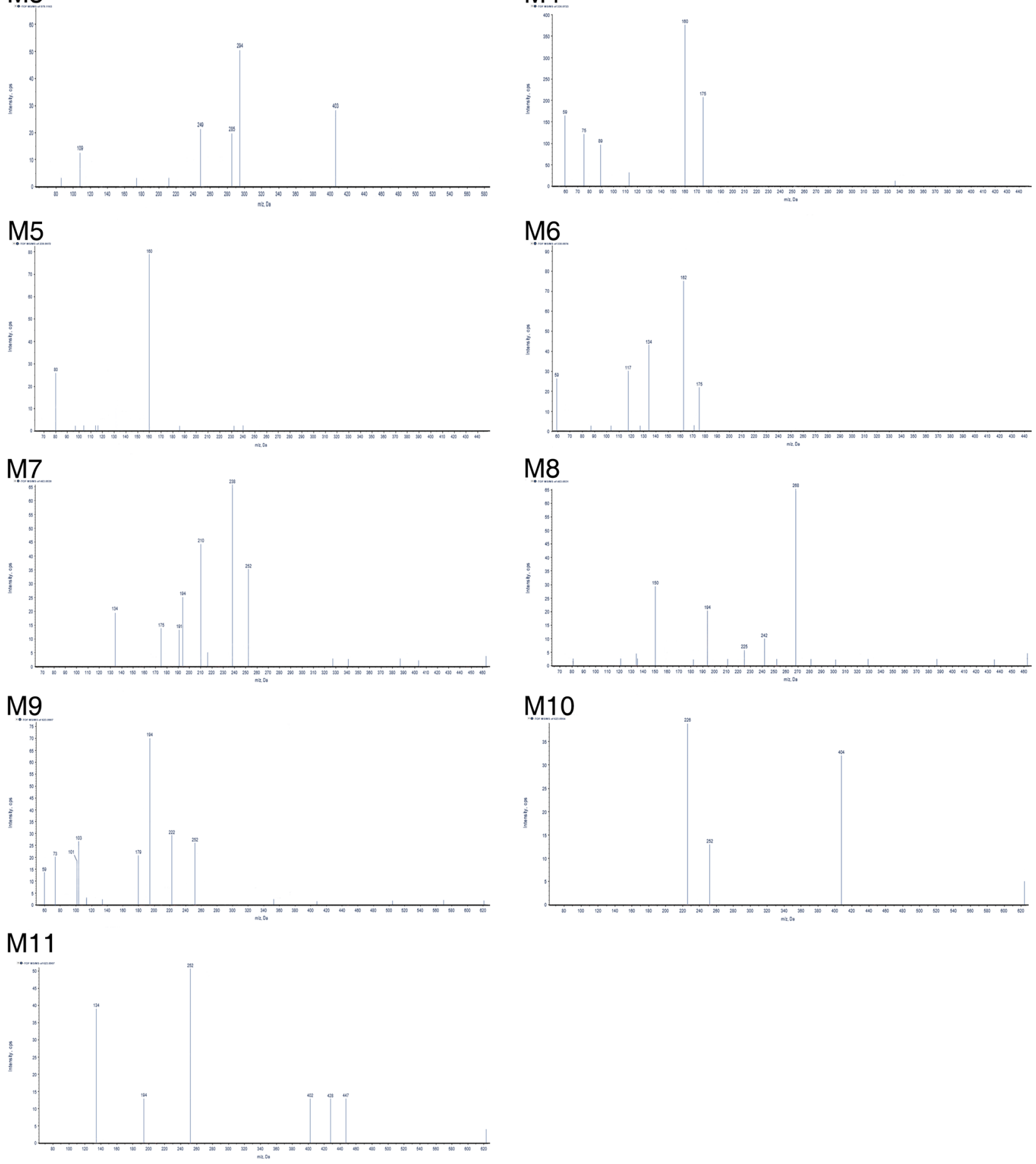

Figure 4: UPLC-MS/MS spectra of metabolites. 
at $\mathrm{m} / \mathrm{z} 252$ and 194 were the same as those of BZG. The fragment ion at $\mathrm{m} / \mathrm{z} 179$ was generated by the loss of $\mathrm{NH}$ (15Da) from the characteristic ion ( $\mathrm{m} / \mathrm{z}$ 194). The fragment ion at $\mathrm{m} / \mathrm{z} 222$ resulted from the addition of CO (28Da) from the fragment ion at $\mathrm{m} / \mathrm{z} 194$. The fragment ions at $\mathrm{m} / \mathrm{z} 59,73,101$, and 103 were formed by the cleavage of the $\mathrm{C}-\mathrm{C}$ or $\mathrm{C}-\mathrm{O}$ bond from the glucuronic acid ion at $\mathrm{m} / \mathrm{z}$ 179. Therefore, M9 were identified as monoglucuronide conjugate of $\mathrm{BZG}$.

The main fragment products of $\mathrm{M} 10$ were at $\mathrm{m} / \mathrm{z}$ 252 and 226, identical to the fragment ions of BZG. The fragment ion at $\mathrm{m} / \mathrm{z} 404$ was generated by the addition of glucuronic acid (176Da) and subsequent loss of one chlorine atom (36Da), $\mathrm{CF}_{3}(69 \mathrm{Da})$ and $\mathrm{C}_{5} \mathrm{H}_{3} \mathrm{~N}_{4}$ (119Da) from BZG. On the basis of the above analysis, M10 was identified as monoglucuronide conjugate of BZG with the site of glucuronidation on part A.

The fragment ion at m/z 447 of M11 was due to a neutral loss of $176 \mathrm{Da}$. The major fragment ions at $\mathrm{m} / \mathrm{z}$ 134, 194, and 252 were the same as that of BZG. In addition, the fragment ion at $\mathrm{m} / \mathrm{z} 428$ was formed by the addition of glucuronic acid (176Da) to the characteristic fragment ion at $\mathrm{m} / \mathrm{z} 252$. Further, loss of CO (28Da) from the fragment ion at m/z 428 generated the fragment ion at $\mathrm{m} / \mathrm{z} 402$. Thus, the site of glucuronidation in M11 was on part B.

\section{Metabolism of BZG in human recombinant enzymes}

A screening of the Cytochrome P450 (CYP) enzymes confirmed that hCYP1A2, 2B6, 2C19, and 2C8 exhibited hydroxylation activity (Table 2). Furthermore, hCYP2B6 exhibited relatively higher metabolic capacity than other CYPs. Further, experiments with recombinant human UDP- glucuronosyltransferases (hUGTs) indicated that UGT1A9 played a major role in the glucuronidation of BZG in HLMs (Table 2).

\section{Virtual screening}

Based on eHiTS analyses, the tested compounds potently inhibited VEGFR-2 (Figure 5). The dock scores of the metabolites and BZG are shown in the Table 3. Five metabolites M2-3, and M9-11 showed favourable eHiTS energy scores. The eHiTS energy scores of M3 (-9.41), M11 (-7.66), M10 (-7.19), M2 (-7.03) and M9 (-6.86) were lower than sorafenib (-6.66). The M3 metabolite was the most suitable for further experiments because of its better ligand efficiency.

\section{DISCUSSION}

An integral part of drug discovery and development is the identification of metabolites of drug formed during phase I and phase II metabolic reactions.
These metabolites may either demonstrate intrinsic pharmacological activity or display specific toxicity. Previous in vitro and in vivo studies showed that sorafenib underwent oxidative metabolism by CYP3A4 and glucuronidation by UGT1A9 into 8 metabolites [16]. In this study, BZG underwent metabolism in phase I that included monohydroxylation (M7, M8) and N-hydroxylation (M1). This indicated that during phase I, hydroxylation of BZG was the major metabolic event in rats. Successive metabolic processes, including sulfonation and degradation (M5), glucuronidation and degradation (M2-4), and acetylation and degradation (M6) were also detected. M4-M6 metabolites were more abundant than others indicating that glucuronidation and degradation were the major metabolic reactions for BZG in rats. The proposed metabolic pathways of BZG are depicted in Figure 3. BZG and its degradation metabolites predominantly passed in the feces, whereas a portion of BZG metabolites were broken down by bacteria flora in the intestine. The intestinal tract plays an important role in the metabolic disposition of BZG and studies are necessary to investigate the intestinal bacteria involved in this process. BZG and its metabolites were excreted more through feces than urine. Therefore, renal elimination is not a major player in BZG clearance.

Furthermore, HLMs and recombinant enzymes are useful in vitro tools for predicting drug metabolic pathways and potential clinical drug-drug interactions. Our experiments with human liver microsomes and recombinant enzymes indicated that BZG underwent oxidative metabolism by hCYP1A2, 2B6, 2C19, and 2C8 (M1) and hCYP3A4 (M7) and glucuronidation by hUGT1A9 to M9. BZG had three potential glucuronidation sites and generated different products in association with UGTs. Our data also indicated that hUGT1A9 was the most efficient enzyme among all UGTs. In humans, hUGT1A9 is predominantly expressed in the liver [17]. Since many drugs are either inhibitors or inducers of hUGT1A9 [18], drug-drug interactions may occur through this pathway. There efficacy of the newly identified phase I and II metabolites needs to be further investigated.

UPLC/QTOF MS has become the cornerstone in drug metabolite identification because of its sensitivity and ability to analyze complex mixtures [19, 20]. In addition, the metabolitepilot ${ }^{\mathrm{TM}} 1.5$ software combined with neutral loss filtration and mass defect filtration technique helps identify multiple bioactive metabolites in vivo with short data interpretation time and high quality structural information. The results provided helpful chemical information to investigate further mechanistic aspects regarding the pharmacological role of BZG.

VEGF family members are the major growth factors that regulate HCC progression [21-23]. Preliminary data has shown that tyrosine kinase inhibitors of VEGFR2 such as sunitinib, sorafenib, and foretinib have high efficacy in HCC patients [15]. In this study, we described 
Table 2: Metabolites of BZG in recombinant human cytochrome P450 enzymes and UDPglucuronosyltransferase enzymes

\begin{tabular}{|c|c|c|c|c|c|c|c|c|}
\hline & \multicolumn{8}{|c|}{ recombinant human cytochrome $\mathbf{P 4 5 0}$ enzymes } \\
\hline & CYP1A2 & CYP2B6 & CYP2C8 & CYP2C9 & CYP2C19 & CYP2D6 & CYP2E1 & CYP3A4 \\
\hline \multirow[t]{3}{*}{ Metabolites } & M1 & M1 & M1 & ND & M1 & ND & ND & M7 \\
\hline & \multicolumn{8}{|c|}{ recombinant human UDP-glucuronosyltransferase enzymes } \\
\hline & UGT1A1 & UGT1A3 & UGT1A6 & UGT1A7 & UGT1A9 & UGT2B7 & UGT2B15 & \\
\hline Metabolites & ND & ND & ND & ND & M9 & ND & ND & \\
\hline
\end{tabular}

Note: ND: No detection.

the computational model of the binding of BZG and its metabolites with the target protein VEGFR2 using docking methods. eHiTS has a novel flexible ligand docking method that exhaustively generates conformations and avoids severe steric clashes between receptor and ligand. Since it is a deterministic system, the results are scientifically reproducible [24]. Our studies showed that metabolites M2-3, and M9-11 efficiently inhibited the binding of VEGFR2. Further in vivo and in vitro are needed to follow-up on our findings.

In conclusion, 11 metabolites (M1-M11) of BZG were identified during phase I and phase II metabolic processes. Of these metabolites, M2-3, and M9-11 efficiently inhibited the binding of VEGFR-2. Our results suggested that the $\mathrm{BZG}$ and its metabolites were effective antagonists of the VEGF/VEGFR2-stimulated angiogenesis and potential candidates for further optimization. Our results provided deeper understanding regarding the metabolism of BZG and useful information regarding the safety and efficacy of BZG.

\section{MATERIALS AND METHODS}

\section{Reagents}

Trisodium isocitric acid, isocitric dehydrogenase, $\beta$-NADP and its reduced form $(\beta$-NADPH), UDPglucuronic acid (UDPGA), and $\beta$-glucuronidase were purchased from Sigma-Aldrich (St. Louis, MO, USA). $S$-Adenosyl- $L$-methionine $p$-toluene sulfonate salt was obtained from Aladdin Reagent (Shanghai, China; purity $>80 \%$ ). Calcium chloride, epsom salt, dipotassium
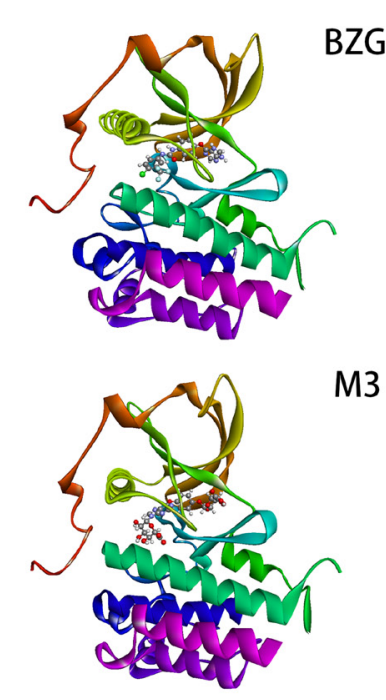

M3

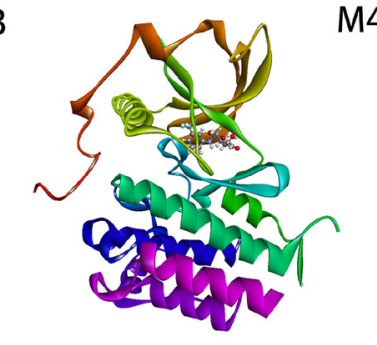

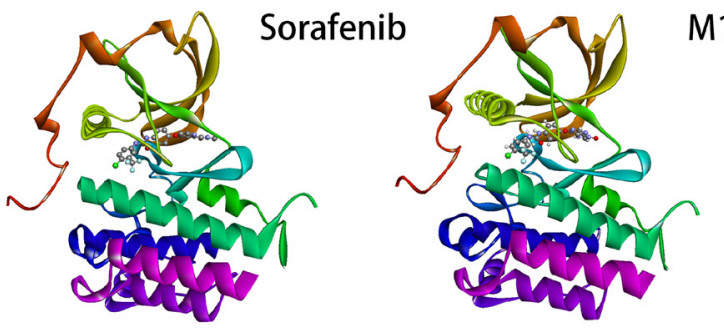

M4

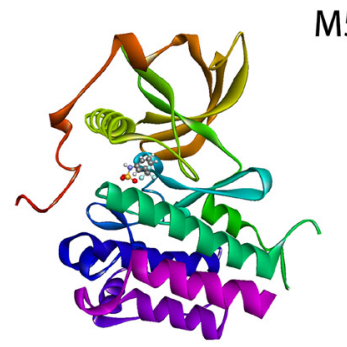

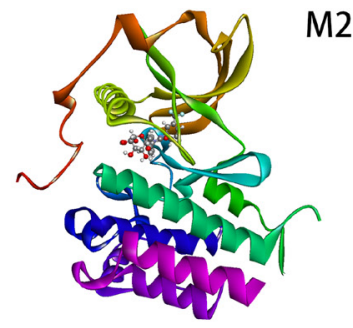

M5

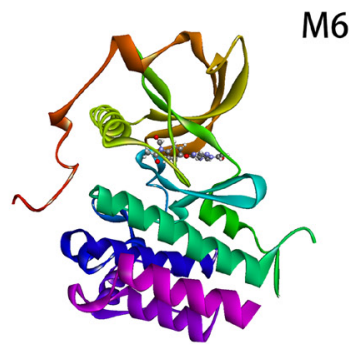

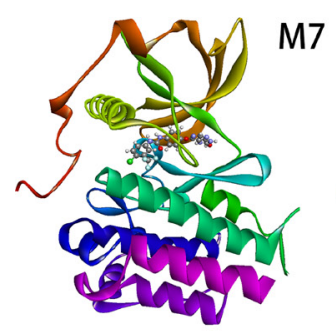
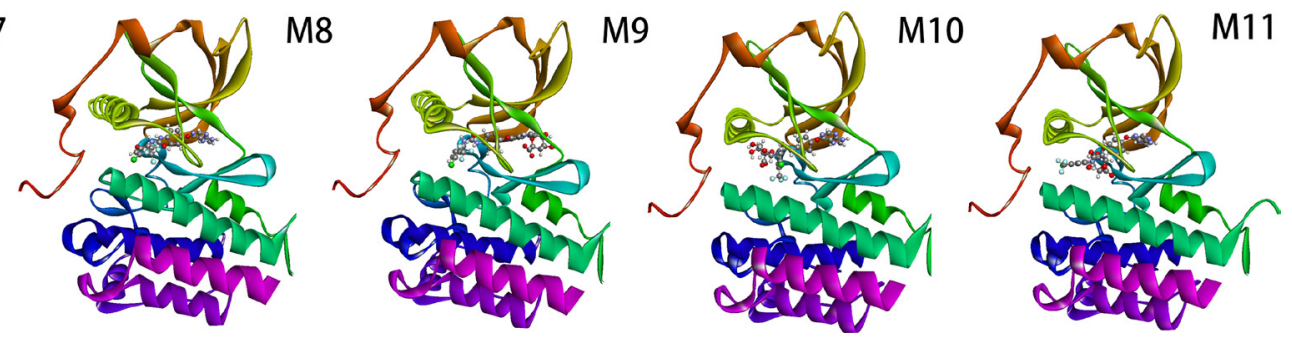

Figure 5: The eHiTS predicted binding model of BZG and its metabolites to VEGFR-2 active site. 
Table 3: eHiTS energy scores of BZG and its metabolites for ligand binding affinities with VEGFR-2 (Sorafenib was used as a positive control)

\begin{tabular}{cc}
\hline Name & eHiTS-Score \\
\hline BZG & -3.711 \\
M1 & -3.471 \\
M2 & -7.034 \\
M3 & -9.412 \\
M4 & -5.459 \\
M5 & -5.005 \\
M6 & -6.272 \\
M7 & -3.507 \\
M8 & -3.466 \\
M9 & -6.861 \\
M10 & -7.19 \\
M11 & -7.658 \\
sorafenib & -6.655 \\
\hline
\end{tabular}

hydrogen phosphate, potassium dihydrogen phosphate, sodium bicarbonate and sodium chloride were supplied by Sinopharm Chemical Reagent (Beijing, China). Tryptone, yeast extract, and cysteine hydrochlorate were obtained from BBI (Canada). All other chemicals were from standard commercial sources and were of the highest quality.

\section{Generation of in vivo BZG metabolites}

BZG was synthesized by Shanghai Medicilon Inc. (Shanghai, China). BZG solution was prepared by dissolving the drug in a mixture of dimethyl sulfoxide:PEG 400:physiological saline in a 1:6:13 ratio by volume. Briefly, the drug was weighed and vortex mixed in a $1.5 \mathrm{ml}$ tube with DMSO until the BZG dissolved followed by addition of PEG 400 and physiological. The solution was clear and no particulate matter was observed.

For in vivo experiments, male Sprague-Dawley rats (200-220 g) were obtained from the Animal Center of Zhejiang Academy of Medical Sciences (Hangzhou, China) and bred in a breeding room with temperature at $25^{\circ} \mathrm{C}, 50 \pm 10 \%$ humidity, and a $12 \mathrm{~h}$ dark-light cycle. They had free access to water and rodent chow all the time. All the experiment animals were acclimatized in the above conditions for one week and fasted overnight before the experiments. The study was approved by the Animal Ethics Committee of Zhejiang University.

Then, twelve rats that were divided into two random groups were treated with $20 \mathrm{mg} \cdot \mathrm{kg}^{-1}$ BZG by oral gavage after an overnight fasting period. After administration, the urine and feces samples were collected from the metabolic cage from one group and bile samples were collected via the bile duct catheter from another group for $24 \mathrm{~h}$. Rats were then euthanized with $\mathrm{CO}_{2}$ after completion of the studies. All samples were stored at $-80^{\circ} \mathrm{C}$ until further analysis.

\section{Preparation of blood, urine, fecal and bile samples for UPLC/Q-TOF/MS analysis}

Blood samples $(300 \mu \mathrm{L})$ were collected into heparinized tubes by scissoring rat tails at 0 (predose), $0.5,3,8,12$, and $48 \mathrm{~h}$ after administration orally. After plasma collection, each blood sample was immediately centrifuged at $4000 \times \mathrm{g}$ for $5 \mathrm{~min}$ at $4^{\circ} \mathrm{C}$, and the samples were stored at $-20^{\circ} \mathrm{C}$ until analysis. Before analysis, the plasma samples were thawed to room temperature and $100 \mu \mathrm{L}$ was vortexed with $200 \mu \mathrm{L}$ acetonitrile in a $1.5 \mathrm{~mL}$ centrifuge tube for $1 \mathrm{~min}$. Subsequently, the mixture was centrifuged at $13,000 \times \mathrm{g}$ for $20 \mathrm{~min}$ at $4^{\circ} \mathrm{C}$. The proteinfree supernatant was separated and a volume of $100 \mu \mathrm{L}$ was pipetted into another $1.5 \mathrm{~mL}$ centrifuge tube and used for analysis.

A mixture of $1 \mathrm{ml}$ urine sample and $1 \mathrm{ml}$ acetonitrile was mixed by vortexing for $5 \mathrm{~min}$ after filtration. The supernatant was transferred into another test tube and evaporated to dryness under vacuum at room temperature. Finally, the residue was reconstituted in $100 \mu \mathrm{L}$ incipient mobile phase by vortexing for $4 \mathrm{~min}$ and further centrifuged at 13,000 rpm for $20 \mathrm{~min}$. The supernatant was used for analysis.

The fecal samples were twice extracted ultrasonically with $3 \mathrm{ml} \cdot \mathrm{g}^{-1}$ methanol for $30 \mathrm{~min}$. The combined methanol extracts were concentrated to nearly $1.0 \mathrm{ml}$ under vacuum and centrifuged at 13,000 rpm for $20 \mathrm{~min}$. The supernatant was stored at $-80^{\circ} \mathrm{C}$ until analysis. The bile sample was prepared similar to the urine samples except that the extraction solution was 1:1 methanol: $\mathrm{H}_{2} \mathrm{O}$ by volume. 


\section{Generation of in vitro metabolites}

\section{Phase I metabolism of BZG in human liver microsomes}

Human liver microsomes were purchased from the Research Institute for Liver Diseases (Shanghai) Co. Ltd. For optimization of the incubation conditions, the linearity of metabolite formation with time (10-120 min) and protein $\left(0.2-2 \mathrm{mg} \cdot \mathrm{ml}^{-1}\right)$ in HLMs was evaluated in advance. All incubation mixtures in a $100 \mu \mathrm{L}$ total volume containing $0.1 \mathrm{M}$ Tris- $\mathrm{HCl}$ (pH 7.4), $15 \mathrm{mM} \mathrm{MgCl}_{2}$, the $\mathrm{NADPH}$-generating system, human liver microsomes $\left(0.5 \mathrm{mg} \cdot \mathrm{ml}^{-1}\right)$, and $100 \mu \mathrm{M}$ BZG were preincubated at $37^{\circ} \mathrm{C}$ for $3 \mathrm{~min}$. The reaction was initiated by adding NADP and NADPH at $37^{\circ} \mathrm{C}$ and stopped after $30 \mathrm{~min}$ by the addition of $300 \mu \mathrm{L}$ of ice-cold acetonitrile. After centrifugation at $13000 \mathrm{rpm}$ for $10 \mathrm{~min}$ ), the supernatant was used for analysis. Control incubations with inactive HLMs or without cofactors were also conducted in parallel.

\section{Phase II metabolism of BZG in human liver microsomes}

Glucuronidation was investigated by incubating HLMs $\left(0.5 \mathrm{mg} \cdot \mathrm{ml}^{-1}\right)$ at $37^{\circ} \mathrm{C}$ for $120 \mathrm{~min}$ in a $100 \mu \mathrm{l}$ medium containing $0.1 \mathrm{M} \mathrm{K}_{2} \mathrm{HPO}_{4}$ buffer, $25 \mu \mathrm{g} \cdot \mathrm{ml}^{-1}$ alamethicin, $10 \mathrm{mM} \mathrm{MgCl}, 50 \mathrm{mM}$ Tris- $\mathrm{HCl}(\mathrm{pH}$ 7.4), and $100 \mathrm{mM}$ BZG. Then $20 \mu \mathrm{L}$ of $4.5 \mathrm{mM}$ UDPGA solution was added to the reaction mixture. The reaction was terminated by the addition of $300 \mu \mathrm{L}$ of ice cold methanol. After centrifugation, the supernatant was used for analysis. Controls with inactive HLMs or without cofactors were conducted in parallel.

The mixed incubation of oxidative and glucuronidation metabolism was investigated in $100 \mu \mathrm{L}$ of buffer containing $0.1 \mathrm{M}$ Tris- $\mathrm{HCl}$ ( $\mathrm{pH} 7.4), 15 \mathrm{mM} \mathrm{MgCl}$, $12 \mathrm{mM}$ dl-isocitrate trisodium, 0.08 unit of isocitrate dehydrogenase, $112.5 \mu \mathrm{g} / \mathrm{ml}$ Triton X-100, $1 \mathrm{mg} \cdot \mathrm{ml}^{-1}$ human liver microsomes, and approximately $200 \mu \mathrm{M}$ BZG in DMSO ( final concentration of DMSO did not exceed $1 \%$ ). The mixture was preincubated at $37^{\circ} \mathrm{C}$ for $3 \mathrm{~min}$. Then $2 \mu \mathrm{L}$ of NADP/NADPH solution and $4 \mu \mathrm{L}$ of UDPGA solution were added to the reaction mixture, and incubation was performed at $37^{\circ} \mathrm{C}$ for $30 \mathrm{~min}$. The reaction was stopped by addition of $300 \mu \mathrm{L}$ of ice cold methanol. After centrifugation, the supernatant was analyzed by UPLC/QTOF MS.

\section{Generation of in vitro metabolites of BZG with recombinant enzymes}

Recombinant human CYP supersomes (CYP1A2, 2B6, 2C8, 2C9, 2C19, 2D6, 2E1 and 3A4) and UGT supersomes (UGT1A1, 1A3, 1A6, 1A7, 1A9, 2B7, and 2B15) expressed in baculovirus-infected insect cells were purchased from BD Biosciences. Experiments to determine in vitro metabolism of BZG with recombinant enzymes was conducted according to the manufacturer's instructions. Briefly, eight human CYP supersomes (CYP1A2, 2B6, 2C8, 2C9, 2C19, 2D6, 2E1, and 3A4) and seven commercially available human UGT supersomes (UGT1A1, 1A3, 1A6, 1A7, 1A9, 2B7, and 2B15) were evaluated for their ability to metabolize $100 \mu \mathrm{M}$ BZG. The incubation conditions were similar to those used with HLMs except that the CYP content was $50 \mathrm{pmol} \mathrm{CYPmL}^{-1}$ and the UGT concentration was $0.5 \mathrm{mg}$ proteinmL ${ }^{-1}$.

\section{Virtual screening of BZG, its metabolites and their docking with VEGFR-2}

The virtual screening of protein-ligand interactions of BZG and its metabolites (Sorafenib was used as a positive control) with VEGFR-2 as the target protein was performed using eHiTS 14.0 from SimBioSys Inc. While eHiTS (http://www.simbiosys.com/ehits) [24, 25] was used for the active site detection and docking, Open Babel (http://openbabel.org) was used for manipulating the ligands chemical formats and acquiring the ligands 3D structures. The scoring was according to the eHiTSScore that is included in the eHiTS software package. The crystal structure of VEGFR-2 in complex with sorafenib (PDB code: 1AH3; http://www.rcsb.org/pdb/) was selected for molecular docking with BZG and its metabolites. However, no special preparation of the 3D structures was applied since eHiTS automatically evaluates all the possible protonation states for the ligands and enzymes. Active site detection was carried out using the 'complex' parameter. The eHiTS program automatically detected the ligand in the complex and selected the part of target protein within a $7 \AA$ margin around the ligand to be the active site. The compound was then docked into the active site using the highest accuracy mode of docking (accuracy parameter was set to 6).

\section{UPLC and MS conditions}

The AB Triple TOF $5600^{\text {plus }}$ System (AB SCIEX, Framingham, USA) was connected to the UPLC system (Waters, Milford, MA, USA) via an electrospray ionization (ESI) interface. Chromatography was performed on the UPLC with a conditioned autosampler at $4^{\circ} \mathrm{C}$ using the Acquity BEH $\mathrm{C}_{18}$ column $(50 \mathrm{~mm} \times 2.1 \mu \mathrm{m}$., $1.7 \mu \mathrm{m}$ particle size;Waters, Milford, MA, USA). The mobile phases were $0.1 \%$ formic acid: $5 \mathrm{mM}$ ammonium formate:water (A) and $0.1 \%$ formic acid: $5 \mathrm{mM}$ ammonium formate:acetonitrile (B). The linear gradient elution of 0-1 min, 5\% B; 1-12 min, 40\% B; 12-16 min, 40-70\% $\mathrm{B} ; 16-18 \mathrm{~min}, 70-95 \% \mathrm{~B} ; 18-19 \mathrm{~min}, 95 \% \mathrm{~B}$ was used to equilibrate the column. The flow rate was set to $0.4 \mathrm{~mL} \cdot \mathrm{min}^{-1}$ and the injection volume was $5 \mu \mathrm{L}$. The optimal MS conditions were: negative ion mode; source 
voltage was $-4.5 \mathrm{kV}$; the source temperature was $550^{\circ} \mathrm{C}$; the pressures of gas1 (Air) and gas2 (Air) were set to 50 psi; the pressure of curtain Gas $\left(\mathrm{N}_{2}\right)$ was set to 35 psi; maximum allowed error was \pm 5 ppm; declustering potential (DP) was $100 \mathrm{~V}$; collision energy (CE) was $10 \mathrm{~V}$. For MS/MS acquisition mode, the parameters were almost the same except that the collision energy (CE) was set at $40 \pm 20 \mathrm{~V}$, ion release delay (IRD) was set at 67 and ion release width (IRW) was set at 25 .

For the 8 most intense metabolite ions, the IDA based auto $\mathrm{MS}^{2}$ was performed in a full cycle scan of $1 \mathrm{~s}$ ). The scan range of $\mathrm{m} / \mathrm{z}$ for the precursor and product ions was set at 100-2000 Da and 50-1500 Da, respectively. The exact mass calibration was performed by the automated calibration delivery system, automatically before each analysis.

HPLC grade acetonitrile, methanol and formic acid were purchased from TEDIA Inc. (Fairfield, USA). Ultrapure water (18.2 M $\Omega$ ) was obtained from an ELGA-Pure lab Ultra system (High Wycombe, UK).

\section{Data processing}

Mass spectral data processing of extracted ion chromatograms and calculation of the elemental compositions using potential metabolite ions was carried out using the PeakView 2.0 software. MetabolitePilot ${ }^{\mathrm{TM}} 1.5$ software was used to automatically identify metabolites by comparing the sample with the controls. Binding affinities were predicted by the docking program eHiTS and a scoring function for the calculation of ligand binding affinities.

\section{Abbreviations}

UPLC/Q-TOFMS: ultra-performance liquid chromatography coupled with quadrupole time-of-flight mass spectrometry; eHiTS: electronic high-throughput screening; HCC: Hepatocellular carcinoma; VEGFR: Vascular endothelial growth factor receptors; PDGF: platelet-derived growth factor; bFGF: basic fibroblast growth factor.

\section{Authors'contributions}

The study was conceptualized and designed by Yan Lou and Yunqing Qiu. Data acquisition was performed by Zhe $\mathrm{Wu}$. The data analysis and interpretation was by Yan Lou, Wenqi Qiu and Qian Wang.The article was drafted by Yan Lou and Wenqi Qiu. The final version was approved by Yunqing Qiu and Su Zeng.

\section{ACKNOWLEDGMENTS}

The study was supported by Science and Technology Department of Zhejiang Province (2016C33066) and
Zhejiang Province Science and Technology of Traditional Chinese Medicine Project (2015ZB025, 2016ZB081).

\section{CONFLICTS OF INTEREST}

The authors declare that they have no conflicts of interest.

\section{REFERENCES}

1. Connell LC, Harding JJ, Abou-Alfa GK. Advanced Hepatocellular Cancer: the Current State of Future Research. Curr Treat Options Oncol. 2016; 17:43.

2. Llovet JM, Villanueva A, Lachenmayer A, Finn RS. Advances in targeted therapies for hepatocellular carcinoma in the genomic era. Nat Rev Clin Oncol. 2015; 12:408-24.

3. Bruix J, Reig M, Sherman M. Evidence-Based Diagnosis, Staging, and Treatment of Patients With Hepatocellular Carcinoma. Gastroenterology. 2016; 150: 835-53.

4. Li Z, Wang B, Tang L, Chen SS, Li J. Quinazoline derivative compound (11d) as a novel angiogenesis inhibitor inhibiting VEGFR2 and blocking VEGFR2-mediated Akt/ mTOR /p70s6k signaling pathway. Iran J Basic Med Sci. 2016; 19:411-6.

5. Wilhelm S, Carter C, Lynch M, Lowinger T, Dumas J, Smith RA, Schwartz B, Simantov R, Kelley S. Discovery and development of sorafenib: a multikinase inhibitor for treating cancer. Nat Rev Drug Discov. 2006; 5:835-44.

6. Qiu YQ, Zhou J, Kang XS, Shen-Tu JZ, Ding LM, Tan FL, Guo J, Li LJ. Discovery of potent, orally active compounds of tyrosine kinase and serine/threonine-protein kinase inhibitor with anti-tumor activity in preclinical assays. Afr J Tradit Complement Altern Med. 2012; 9:431-9.

7. Qiu YQ, Kang XS, Ding LM, Yu W, Tan FL, Guo J, Zhou J, Wang JB, Chen ML, Yang C. In vitro and in vivo study of BZG-4000: Anti-tumoral activity of human hepatocellular carcinoma. Journal of Pure \& Applied Microbiology. 2013; 7:323-8.

8. Qiu YQ, Zhou J, Kang XS, Ding LM, Yu W, Tan FL, Deng DF. Effect of BZG-4000, a novel multi-targeted kinase inhibitor with potent anticancer activity, on a hepatocellular carcinoma xenograft model. Sci Rep. 2014; 4:4324.

9. Lou Y, Wang L, Qian QB, You J, Qiu WQ, Wang Q, Zhu KD, Qiu YQ. Preclinical pharmacokinetics and tissue distribution of a novel multikinase inhibitor BZG by validated UPLC-MS/MS assay. J Pharm Biomed Anal. 2016; 121:107-13.

10. Lathia C, Lettieri J, Cihon F, Gallentine M, Radtke M, Sundaresan P. Lack of effect of ketoconazole-mediated CYP3A inhibition on sorafenib clinical pharmacokinetics. Cancer Chemother Pharmacol. 2006; 57:685-92.

11. Kuehl P, Zhang J, Lin Y, Lamba J, Assem M, Schuetz J, Watkins PB, Daly A, Wrighton SA, Hall SD, Maurel P, 
Relling M, Brimer C, et al. Sequence diversity in CYP3A promoters and characterization of the genetic basis of polymorphic CYP3A5 expression. Nat Genet. 2001; 27:383-91.

12. Cui PH, Rawling T, Gillani TB, Bourget K, Wang XS, Zhou F, Murray M. Anti-proliferative actions of N'desmethylsorafenib in human breast cancer cells. Biochem Pharmacol. 2013; 86:419-27.

13. Carmeliet P. VEGF as a key mediator of angiogenesis in cancer. Oncology. 2005; 69:4-10.

14. Olsson AK, Dimberg A, Kreuger J, Claesson-Welsh L. VEGF receptor signalling - in control of vascular function. Nat Rev Mol Cell Biol. 2006; 7:359-71.

15. Wang W, Ma XP, Shi Z, Zhang P, Ding DL, Huang HX, Saiyin HG, Chen TY, Lu PX, Wang NJ, Yu H, Sun J, Zheng SL, et al. Epidermal growth factor receptor pathway polymorphisms and the prognosis of hepatocellular carcinoma. Am J Cancer Res. 2015; 5:396-410.

16. Shimada M, Okawa H, Maejima T, Yanagi T, Hisamichi K, Matsuura M, Akasaka K, Tsuchiya M, Kondo Y, Shimosegawa T, Mori M, Maekawa M, Suzuki H, et al. A quantitative HPLC-UV method for determination of serum sorafenib and sorafenib N-oxide and its application in hepatocarcinoma patients. Tohoku J Exp Med. 2014; 233: 103-12.

17. Kiang TK, Ensom MH, Chang TK. UDPglucuronosyltransferases and clinical drug-drug interactions. Pharmacol Ther. 2005; 106:97-132.

18. Miners JO, Mackenzie PI, Knights KM. The prediction of drug-glucuronidation parameters in humans: UDPglucuronosyltransferase enzyme-selective substrate and inhibitor probes for reaction phenotyping and in vitro- in vivo extrapolation of drug clearance and drug-drug interaction potential. Drug Metab Rev. 2010; 42:196-208.

19. Lou Y, Zheng JQ, Wang BH, Zhang XG, Zhang X, Zeng S. Metabolites characterization of chamaechromone in vivo and in vitro by using ultra-performance liquid chromatography/Xevo G2 quadrupole time-of-flight tandem mass spectrometry. J Ethnopharmacol. 2014; 151:242-52.

20. Lou Y, Zheng JQ, Hu HH, Lee J, Zeng S. Application of ultra-performance liquid chromatography coupled with quadrupole time-of-flight mass spectrometry to identify curcumin metabolites produced by human intestinal bacteria. J Chromatogr B Analyt Technol Biomed Life Sci. 2015; 985:38-47.

21. Muto J, Shirabe K, Sugimachi K, Maehara Y. Review of angiogenesis in hepatocellular carcinoma. Hepatol Res. 2015; 45:1-9.

22. Carmeliet $P$, Jain RK. Molecular mechanisms and clinical applications of angiogenesis. Nature. 2011; 473:298-307.

23. Scartozzi M, Faloppi L, Svegliati Baroni G, Loretelli C, Piscaglia F, Iavarone M, Toniutto P, Fava G, De Minicis S, Mandolesi A, Bianconi M, Giampieri R, Granito A, et al. VEGF and VEGFR genotyping in the prediction of clinical outcome for HCC patients receiving sorafenib: the ALICE-1 study. Int J Cancer. 2014; 135:1247-56.

24. Zsoldos Z, Reid D, Simon A, Sadjad SB, Johnson AP. eHiTS: a new fast, exhaustive flexible ligand docking system. J Mol Graph Model. 2007; 26:198-212.

25. Zsoldos Z, Reid D, Simon A, Sadjad BS, Johnson AP. eHiTS: an innovative approach to the docking and scoring function problems. Curr Protein Pept Sci. 2006; 7:421-35. 